
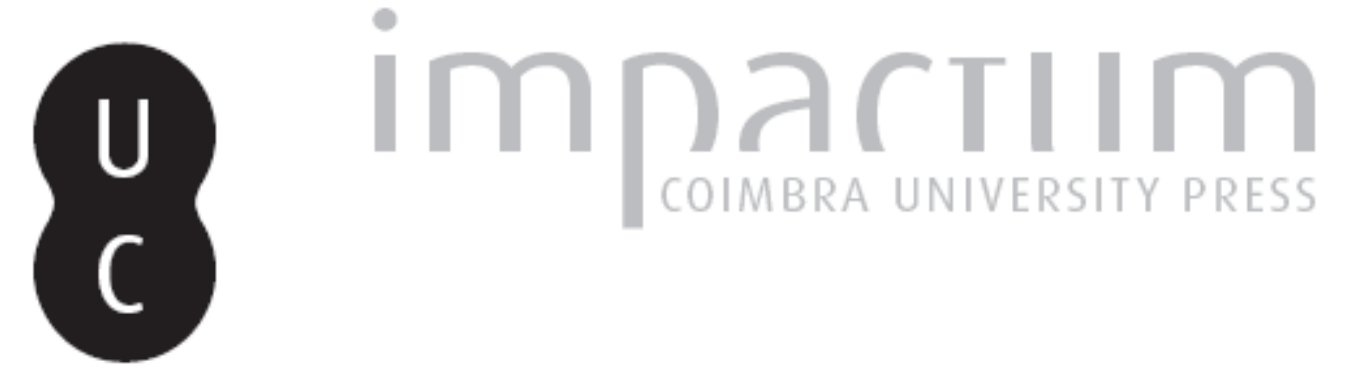

\title{
La colectivización de la asistencia social en la provincia de León a través de los hospitales
}

\author{
Autor(es): $\quad$ Pérez Álvarez, María José \\ Publicado por: Centro de História da Sociedade e da Cultura \\ URL \\ persistente: \\ URI:http://hdl.handle.net/10316.2/39520 \\ DOI: \\ DOI:http://dx.doi.org/10.14195/1645-2259_10-1_10
}

Accessed : $\quad$ 26-Apr-2023 04:55:55

A navegação consulta e descarregamento dos títulos inseridos nas Bibliotecas Digitais UC Digitalis, UC Pombalina e UC Impactum, pressupõem a aceitação plena e sem reservas dos Termos e Condições de Uso destas Bibliotecas Digitais, disponíveis em https://digitalis.uc.pt/pt-pt/termos.

Conforme exposto nos referidos Termos e Condições de Uso, o descarregamento de títulos de acesso restrito requer uma licença válida de autorização devendo o utilizador aceder ao(s) documento(s) a partir de um endereço de IP da instituição detentora da supramencionada licença.

Ao utilizador é apenas permitido o descarregamento para uso pessoal, pelo que o emprego do(s) título(s) descarregado(s) para outro fim, designadamente comercial, carece de autorização do respetivo autor ou editor da obra.

Na medida em que todas as obras da UC Digitalis se encontram protegidas pelo Código do Direito de Autor e Direitos Conexos e demais legislação aplicável, toda a cópia, parcial ou total, deste documento, nos casos em que é legalmente admitida, deverá conter ou fazer-se acompanhar por este aviso.

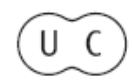




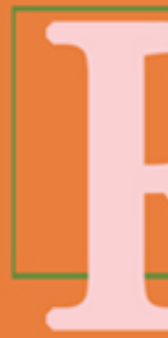

\section{evista de História}

da Sociedade e da

Cultura

\section{Tomo I}

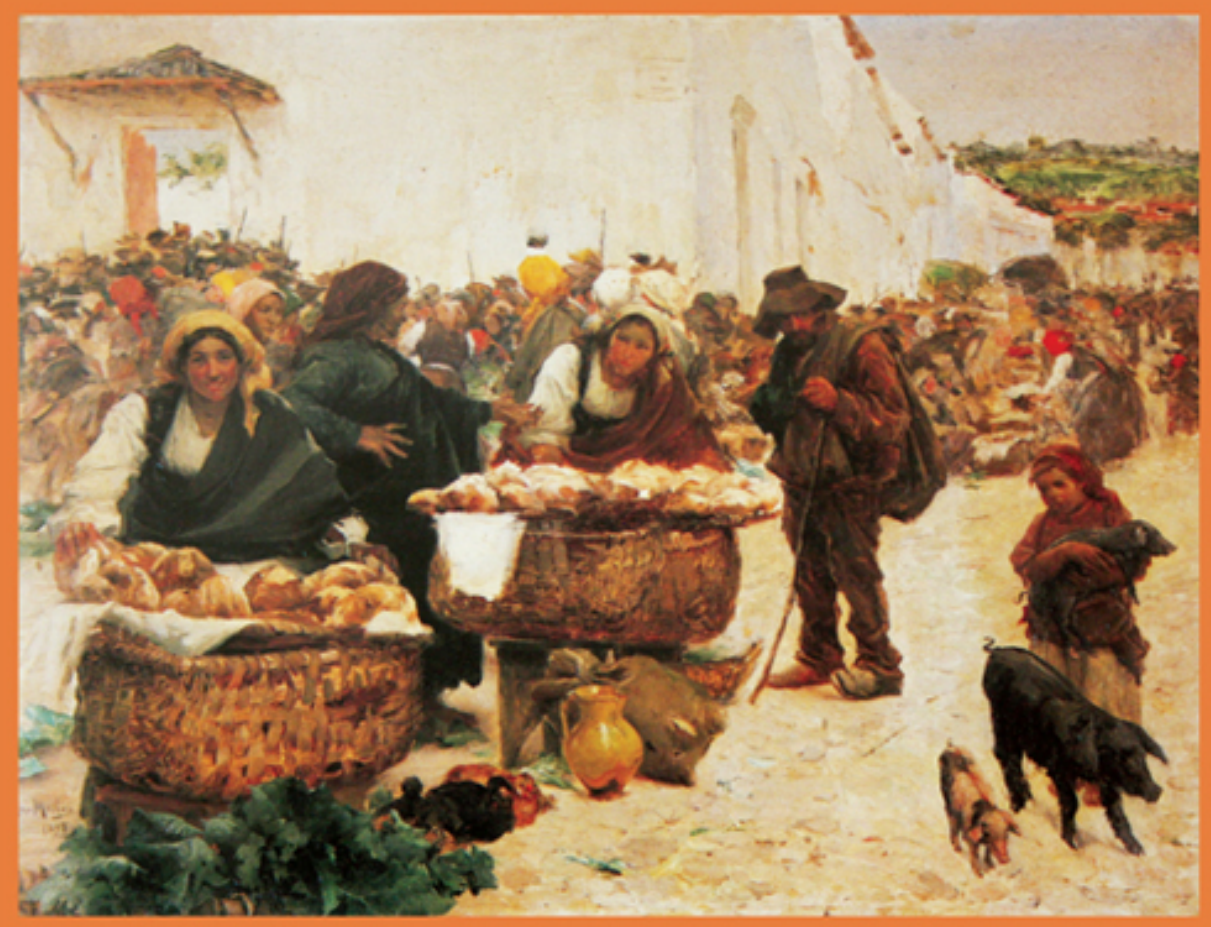

Centro de História da Sociedade e da Cultura Universidade de Coimbra

Coimbra 


\title{
La colectivización de la asistencia social en la provincia de León a través de los hospitales
}

\author{
María José Pérez Álvarez \\ Universidad de León \\ maria-jose.perez@unileon.es \\ Texto recebido em/ Text submitted on: 16/02/2010 \\ Texto aprovado em/ Text approved on: 12/04/2010
}

\section{Resumo/Abstract:}

A comarca de Bierzo foi território de passagem do Caminho de Santiago e à sua sombra foi-se desenhando durante a Idade Média uma importante rede assistencial. Muitos destes estabelecimentos tiveram uma vida efémera, outros, pelo contrário, estiveram activos durante toda a Idade Moderna, como foi o caso de San Juan de Cacabelos ou de Reina de Ponferrada. Contudo, enquanto este, após a sua refundação, se adaptou às novas circunstâncias, convertendo-se num centro médico-assistencial, o primeiro, ancorado num regime assistencial muito primário, foi definhando até praticamente desaparecer.

The district of Bierzo was a stop in the Camino of Santiago and in its shadow an important support network developed in the Middle Ages. Many of these settlements were ephemeral, but others survived until the Early Modern Age, like San Juan de Cacabelos or Reina de Ponferrada. However, while the latter was reconstructed and adjusted to the new surroundings, becoming a health care and assistance-providing centre, the former, anchored in a rather primary scheme of assistance, gradually shrivelled to near extinction.

Palavras chave/Keywords:

León; El Bierzo; Hospital; Idade Moderna.

León; El Bierzo; Hospital; Modern Age. 
Durante la Edad Media ${ }^{1}$ el término hospital se utilizó para designar a todos aquellos centros que habían nacido con una vocación de asistencia caritativa a los pobres y/o enfermos, independientemente de si ofrecían o no cuidados médicos. Así siguió haciéndose durante el periodo histórico siguiente $^{2}$, en el que, por otra parte, continuó teniendo vigencia el ideal medieval que consideraba la asistencia al pobre como una manera de lograr la salvación espiritual de todos los involucrados en el auxilio hospitalario. De hecho, en las visitas pastorales que hemos podido consultar del hospital de la Reina, en Ponferrada, o de San Juan, en Cacabelos, se recuerda a los administradores que tuvieran presente "que el pobre encarna la figura de Cristo"3.

A lo largo de la Edad Media se fue configurando una importante estructura hospitalaria en el Bierzo, ligada al Camino de Santiago. Durante esa etapa histórica llegaron a estar operativos en torno a medio centenar de centros, dependientes en su mayoría de instituciones religiosas ${ }^{4}$. La mayor parte eran simples refugios, sin más pretensiones que ofrecer pernocta y algún

1 Este trabajo forma parte del proyecto de investigación Cultura popular y mentalidades colectivas en la provincia de León durante la Edad Moderna, financiado por la Diputación Provincial de León.

2 Por ejemplo, con el término hospital designó D. Andrés Pérez de Capillas el establecimiento que fundó en 1537 en la localidad leonesa de Puente Villarente, que era en realidad un mero albergue de peregrinos y transeúntes. AHN Clero, Libro 5.522.

3 Los obispos estaban facultados para realizar visitas en centros de estas características que no eran dependientes del clero. Vid. CARMONA GARCÍA, Juan Ignacio - El sistema de la hospitalidad pública en la Sevilla del Antiguo Régimen. Sevilla: Diputación de Sevilla, 1979, p. 19.

4 Durante la Edad Moderna, y a pesar de que algunos pensadores humanistas, como Luís Vives, recordaron a los monarcas la responsabilidad que debía asumir el poder civil en la secularización de estos centros, en la práctica siguieron muy vinculados a la Iglesia. Los planteamientos de los humanistas perdieron fuerza en el siglo XVII, debido a las dificultades económicas de la hacienda estatal y al clima contrarreformista, dentro del cual la asistencia a los necesitados volvía a ser una pieza fundamental en el ejercicio de la caridad. De nuevo, en el siglo XVIII se vuelve a primar la responsabilidad civil sobre la caridad religiosa a la hora de ofrecer auxilio al necesitado. A pesar de las discrepancias surgidas en la centuria dieciochesca entre los conservadores de la caridad tradicional y los renovadores y de los intentos de Carlos III de secularizar la beneficencia, tampoco en ese momento, ni en los inmediatamente posteriores, se dieron avances importantes en este sentido. Vid. GARCÍA GUERRA, Delfín - El hospital Real de Santiago (1499-1804). La Coruña: Fundación Barrie de la Maza, 1983, p. 48-49 y 138; CALLAHAN, William J. - Caridad, sociedad y economía en el siglo XVIII. Moneda y Crédito. 146 (1978) 65-77; SCHUBERT, Adrián - Nuevos enfoques sobre la beneficencia en España en el siglo XIX. Studia Zamorensia. 19 (1984) 
alimento a los transeúntes. Ese panorama cambió notablemente tras la reestructuración de infraestructura hospitalaria llevada a cabo a comienzos de la Edad Moderna. En el siglo XVI ya habían desaparecido muchos de esos pequeños centros y aún seguiría el desmantelamiento en fechas posteriores. Tal quiebra fue el resultado, en unos casos, de los procesos de fusión promovidos por la Corona desde la época de los Reyes Católicos ${ }^{5} \mathrm{y}$, en otros, de la escasa dotación económica que tenían para subsistir, lo que les llevó a languidecer hasta perder sus funciones.

La ruta jacobea, procedente de la ciudad de Astorga, se internaba en el Bierzo por tres ramales que confluían en Ponferrada. El que atravesaba los Montes de León cruzando por el puerto de Manzanal, otro por el de Foncebadón y el tercero era la denominada "Vía Cerezal". El acceso más transitado era el segundo, por esa razón concentró durante la Edad Media la mayor actividad asistencial. Si bien, la ruta jacobea durante la Edad Moderna ya había perdido gran parte de significado religioso aún conservaba el económico o militar, lo que la convertía en una vía de comunicación de primer orden. De ahí, que en este momento el principal caudal de viandantes ya no fueran peregrinos, sino transeúntes de todas las capas sociales que la recorrían con fines muy diversos ${ }^{7}$. No obstante, ese cambio de sentido de la ruta jacobea no fue óbice para que un número importante de esas

325-336. CARASA SOTO, Pedro - Historia de la beneficencia en Castilla y León. Poder y pobreza en la sociedad castellana. Valladolid: Universidad, 1991, p. 9-16.

5 Sobre la reunificación hospitalaria en la primera mitad del siglo XVI pueden consultarse, entre otros, GARCÍA ORO José y PORTELA SILVA María José - Felipe II y el problema hospitalario: reforma y patronato. Cuadernos de Historia Moderna. 25 (2000) 87-125, p. 89-90; LEÓN VARGAS Milagros -La reducción de los hospitales antequeranos en la primera mitad del siglo XVII, in CORTÉS PEÑA, Antonio Luís LÓPEZ-GUADALUPE Miguel Luís y LARA RAMOS Antonio (Eds.) - Iglesia y Sociedad en el Reino de Granada. Granada: Universidad de Granada, 2003, p. 235-247. Para el Bierzo vd. CAVERO DOMÍNGUEZ Gregoria -Peregrinos e indigentes en el Bierzo Medieval (siglos XI-XVI). Hospitales en el Camino de Santiago. Ponferrada: Basílica de la Encina y Asociación de amigos del Camino de Santiago, 1987.

6 Gregoria CAVERO DOMÍNGUEZ, Peregrinos e indigentes en el Bierzo Medieval.., cit., p. 43-52.

7 En la Edad Moderna este camino perdió parte su vertiente religiosa para dar paso a la limosnera, pasando a convertirse en un "camino de pobres", que buscaban sostenerse a costa de las limosnas hospitalarias. Vid. CARASA SOTO, Pedro - Historia de la beneficencia..., cit., p. 31 . 
gentes continuaran dependiendo del acogimiento caritativo que ofrecían tales hospitales.

Según las "Respuestas Generales del Catastro del marqués de la Ensenada", elaborado en la década de los cincuenta del siglo XVIII, aún quedaban en la comarca berciana quince de los centros asistenciales que habían sido fundados en la época medieval. La excepción sería el de Nuestra Señora de la Regla de Ponferrada, para el que se señala como fecha fundacional 1498, no obstante, parece que se trata de una refundación de la agregación de otros centros anteriores. Excepto tres de esos establecimientos, el resto estaban situados en alguna de las localidades que jalonaban la ruta principal del Camino de Santiago o en otras atravesadas por vías secundarias ${ }^{8}$ (Mapa n. ${ }^{\circ}$ 1). En el primer caso se encontraban el de Manjarín, Molinaseca, Ponferrada, Columbrianos, Cacabelos, Villafranca y Herrerías. Por su parte, Los Barrios, donde había tres centros asistenciales, uno en cada pequeño núcleo que componía la población, formaba parte de la ruta alternativa que surgía entre El Acebo y Molinaseca. Por Bembibre y San Román cruzaba la vía que enlazaba Astorga con el Bierzo por el puerto del Manzanal. En cuanto a San Esteban de Valdueza y Villanueva, que eran dos de las excepciones, se trata de localidades por las que el viajero debía transitar si se encaminaba a Peñalba de Santiago, donde se encuentra el sepulcro del venerado San Genadio. Finalmente, el hospital de Borrenes estaría ligado a la ruta que comunicaba el Bierzo con la provincia de Orense.

Respecto a la capacidad económica de esos centros, la única fuente que nos ofrece una visión de conjunto es el Catastro del marqués de la Ensenada. De acuerdo con esa información, los hospitales más modestos eran los de Villanueva de Valdueza, Manjarín, San Esteban y Columbrianos. Sus ingresos, para "asistir a los pobres que se recogen", oscilaban entre los trece y treinta y ocho reales al año. En un segundo nivel, con una dotación que iba los doscientos a poco más de cuatrocientos, estaban los de Herrerías,

8 La concentración de hospitales, que aún permanecían abiertos en la Edad Moderna, en torno a la ruta jacobea tiene su reflejo en otros territorios por los que atravesaba el camino, caso por ejemplo de La Rioja. Lo cual resulta lógico si tenemos en cuenta que casi todos ellos habían nacido para ese servicio. CARASA SOTO, Pedro - Crisis y transformaciones de la beneficencia en el Antiguo Régimen. Aproximación al sistema hospitalario de la Rioja entre 1750-1907. Cuadernos de Investigación: Historia. 10 (1984) 7-26, p. 12. 


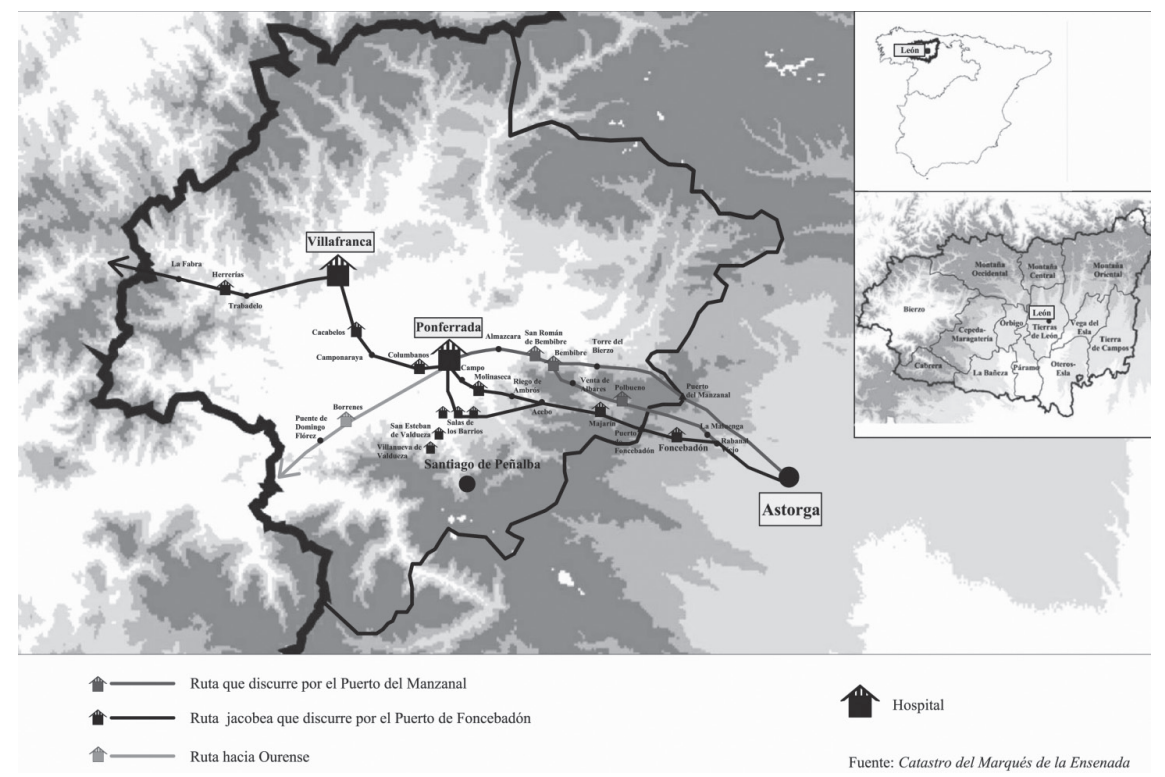

Mapa 1 - La red hospitalaria en la comarca del Bierzo a mediados del siglo XVIII

Bembibre, Borrenes, Villar de Salas y Cacabelos. Concretamente en el de Villar se llegaban a ofrecer a los albergados veinticuatro maravedís al día para cubrir las necesidades de alimento y, además, en caso de necesitar cuidados médicos pagaban al cirujano que los asistiese y las medicinas necesarias. De mayor envergadura eran los de Villafranca del Bierzo y Ponferrada, que contaban con asistencia médico-sanitaria. Finalmente, desconocemos las rentas de los de Molinaseca, Barrios de Salas, Barrio de Lombillo y San Román.

Para acercarnos al modelo hospitalario berciano durante la Edad Moderna tomaremos como referencia dos establecimientos bien distintos: el de la Reina de Ponferrada y San Juan Bautista de Cacabelos, ambos bajo administración laica. El primero era un hospital en el pleno sentido del término, puesto que, sin descuidar las necesidades del alma, contaba con un personal sanitario de plantilla que se encargaba de proporcionar al enfermo las atenciones médico-sanitarias necesarias para su restablecimiento. Por su parte, el de San Juan podríamos catalogarlo como un simple refugio, aunque su dotación económica le permitía ofrecer a los demandantes de auxilio unas prestaciones más completas que otros albergues diseminados por la comarca. 


\section{Las instalaciones hospitalarias}

El hospital de la Reina lo fundaron los Reyes Católicos en 1498, o, también podríamos decir, refundaron, ya que es el resultado de la fusión de una serie de centros más antiguos ${ }^{9}$. Se inserta, por lo tanto, en el marco de la política reestructuradora impulsada por los monarcas renacentistas. El nuevo establecimiento estuvo gestionado desde sus inicios por el ayuntamiento, aunque parece que, en algún momento, también pudo haber existido una cofradía ligada a é $1^{10}$. Se trataba de un centro de dimensiones modestas, pues a finales del siglo XVIII solamente poseía ocho camas para atender a los pobres enfermos de ambos sexos. Por su parte, el de San Juan de Cacabelos, también bajo la administración de la villa, había sido fundado en época medieval y aún era más humilde que el anterior. Los dos formaban parte de pequeños complejos, compuestos por el edificio hospitalario, la capilla o ermita y el cementerio. Además, sabemos que el de Cacabelos poseía una huerta en la que se plantaba "verdura y otras cosas para el servicio de los enfermos"; desconocemos si el de Ponferrada la tenía algo similar, pero teniendo en cuenta su utilidad las probabilidades son elevadas, de lo que sí disponía éste era una botica en una dependencia anexa.

Son muy pocos los datos que hemos logrado reunir de los edificios hospitalarios. La imagen que reflejan las visitas pastorales y los libros de acuerdos de la Reina, es la de unas instalaciones modestas, con importantes carencias estructurales y deficitarias condiciones de salubridad. Por ejemplo, en 1799 los administradores del centro ponferradino dejaron constancia ${ }^{11}$ que el aposento de mujeres "está amenazando ruina" y que la cocina carecía de ventilación. En 1801 era la sala de curación de enfermas la más deteriorada, le achacaban ser el foco de no pocas infecciones. Esta situación, que calificaban de insostenible, llevó a sus rectores a agilizar una petición de

9 Para más información Vid. CAVERO DOMÍNGUEZ, Gregoria - Peregrinos e indigentes..., cit., p. 71-78.

${ }^{10}$ Esa hermandad aparece citada en el documento en el que los Reyes Católicos conceden el "juro" al hospital. Sin embargo ya nada se dice de ella en el reglamento elaborado a finales del siglo XVIII - El hospital de Nuestra señora de Santa María de la Villa de Ponferrada. Estudios Bercianos. 13 (1990), 43-47 (44).

${ }^{11}$ AHMP (Archivo Histórico Municipal de Ponferrada), Libro de Acuerdos del Hospital de la Reina, Leg. 23, Vol. 6. 
licencia de obras al Consejo de Castilla, pero como en años precedentes los proyectos no se concretizaron, manteniéndose las deficiencias ya señaladas. El momento más crítico se produjo como consecuencia de la Guerra de Independencia. De hecho, entre 1808 y 1816 el establecimiento permaneció cerrado por los desperfectos que en él ocasionaron las tropas francesas.

La dotación interna del edificio iba acorde con la precariedad de las instalaciones. En 1566 tenía un escaño, una silla, un arca grande, dos caballetes, un candil de hierro y once lechos de madera; en la cocina se custodiaba una caldera grande y unas "pregancias"; y pobre era también el ajuar de las camas. Tres años después, salvo leves variaciones en el número de sábanas y mantas, todo se mantenía igual. Sería a finales de la década de los setenta del siglo XVII, merced al pequeño patrimonio que se había ido conformando y a la agregación del hospital de San Lázaro, cuando las instalaciones aparezcan algo mejor surtidas. De todas formas, esa situación de bonanza duró muy poco, ya que las alusiones al "desaliño y desabrigo en los pobres enfermos" continuarán siendo frecuentes ${ }^{12}$.

Hasta finales del siglo XVIII la plantilla de personal que dirigía y trabajaba en el hospital ponferradino era muy reducida. El regimiento delegaba la gestión económica en un "mayordomo" o administrador, que a su vez era el superior jerárquico del resto de trabajadores del hospital. Por su parte, la atención a los enfermos estaba a cargo de un médico ${ }^{13}$, un sangrador, un capellán y el hospitalero ${ }^{14}$. Este organigrama tan simple se complicó considerablemente tras la aprobación de un nuevo reglamento ${ }^{15}$ en

${ }^{12}$ Los inventarios aparecen intercalados entre los libros de cuentas. AHMP Libros de cuentas del Hospital de la Reina.

${ }^{13}$ El que, de una forma u otra, se facilitara cobertura médica a todas las capas sociales implicó una socialización de la medicina. Es conveniente recordar que sólo los más pudientes podían tener acceso a un tratamiento impuesto por un titulado universitario. Vid. GARCÍA GUERRA, Delfín - El hospital Real de Santiago..., cit., p. 58-59, y La asistencia hospitalaria en la España Moderna: el Hospital Real de Santiago. Estudios de Historia Social. 7 (1978) 289-327.

${ }^{14} \mathrm{La}$ composición de la plantilla del centro es muy similar a la de otros hospitales de mayor envergadura como, por ejemplo, el de Loja, con capacidad para 24 camas. Vid. CORONAS TEJADA, Luís - El hospital Real de Nuestra Señora de la Misericordia de Loja. Granada: Universidad de Granada, 1990, pp. 46 y ss.

${ }^{15}$ Para su elaboración se tomó como modelo un reglamento que les había enviado la Cámara de Castilla. Con esas modificaciones pretendían subsanar el "deplorable estado y mala administración de las rentas” del centro. AHMP Leg. 26 vol. 3. 
1788. A partir de entonces el Hospital de la Reina pasaría a estar gestionado por una junta, formada por nueve personas: el corregidor de la villa, sobre el que recaía la presidencia, y ocho vocales. Una vez constituida aquella se nombraban trece cargos para desempeñar diferentes funciones. Cinco de ellos recaían sobre los vocales, concretamente el de secretario y cuatro "comisarios". Dos de esos "comisarios" se centrarían en la gestión de la dehesa perteneciente al centro, uno a la botica y otro a la caridad. Los puestos restantes - c apellán, "mayordomo", médico, cirujano, "regente de botica", practicante, enfermera y portero - se cubrían con personal externo a la directiva.

Respecto al hospital de San Juan ${ }^{16}$, de la visita realizada en 1752 se desprende que el edificio se distribuía en tres salas, una con capacidad para 4 hombres, otra para dos mujeres y una enfermería. Como en el caso anterior, son constantes las referencias al mal estado del edificio, pero en éste, además, también se señalaba la falta de aseo en las instalaciones - no así la capilla generalmente "limpia y aseada" - y de respeto al cementerio. En 1748 las deficiencias estructurales eran tan graves que, de no acometerse una reforma rápida del edificio, la pared maestra peligraba "derrumbe"17. No menos preocupantes eran las condiciones de salubridad y de dotación. Apenas llegaba la luz a la enfermería y dormitorios, por lo que tampoco podrían ventilarse adecuadamente $\mathrm{y}$, asimismo, había escasez de "camas y abrigo". Lo que más lamentaba el visitador era que a esa situación no se había llegado por falta de fondos, sino por desidia, pues los mayordomos estaban debiendo un total de 5.435 reales de los alcances de años precedentes. Ordenaba que se recaudasen esas cantidades para invertirlas en el remozado del inmueble y en la adquisición del ajuar necesario. Las lagunas que la contabilidad tiene por esos años no nos han permitido saber si tales obras de construcción se llevaron a cabo. No obstante, sí que podrían haberse hecho

${ }^{16}$ AHDA (Archivo Histórico Diocesano de Astorga), Libro de Cuentas del Hospital de San Juan V.2. Se conservan dos libros del citado hospital, el primero (V) comienza en 1574 y finaliza en 1655 y el V.2 va de 1655 a 1733. En ellos, con abundantes lagunas por el medio, se anotaron las cuentas, las vistas, inventarios...

17 “....teniendo en cuenta el infeliz y deplorable estado a que se halla reducido el hospital y considerando que la razón, justicia y piedad no permiten que los pobres peregrinos y enfermos apenas encuentren en él una pocas pajas en que recostarse y que su edificio está tan ruinoso como manifiesta la visita..". AHDA Libro de San Juan. 
algunos pequeños arreglos, puesto que en la visita siguiente, la de 1752, sólo se hace referencia a cuestiones relacionadas con el menaje. Pero el hecho de que en 1767 y 1773 se reincida en el tema de las deudas contraídas con el hospital por los sucesivos mayordomos, que cada vez iban incrementándose más, nos conduce a pensar que en ningún momento se reunió la cantidad de dinero necesaria para acometer las reformas estructurales necesarias para su total reconstrucción.

En lo referente al cementerio, si bien en el siglo XVIII no encontramos alusiones a él, en la centuria anterior las visitas proyectan una imagen de abandono absoluto, por acumulación de basuras, e incluso profanación. En 1649 el licenciado D. Santiago Méndez ordenaba al mayordomo que recogiera los huesos y las calaveras que estaban esparcidos por el cementerio y que cavara una sepultura "común" sobre la que debería de colocar una cruz. Cinco años después mandaba que se levantara una pared de piedra en torno al camposanto. No sabemos si el recinto llegó a cerrarse correctamente, pero la persona que realizó la visita de 1670 se encontró a un cerdo pastando en él.

Como en el caso del establecimiento ponferradino, ese abandono exterior del edificio y sus anexos se proyectaba en el interior. En 1623 la dotación del hospital era pésima, el ajuar lo componían cuatro mantas viejas y otra desgarrada, tres colchones usados, un cobertor, una sábana, un paño y un cabezal viejo. Otro tanto puede decirse del mobiliario, disponían de 5 lechos sin fondo, un arca y un banco. Además de lo señalado poseían una "gramallera", para colgar el pozal de cobre en el que se hacía el potaje, y una pala de hierro muy deteriorada para cavar las sepulturas. Diez años después se realizó un nuevo recuento en el que se aprecia como la dotación había ido aumentando por las nuevas adquisiciones ordenadas en $1625^{18}$ y las mandas testamentarias ${ }^{19}$ recibidas. Pero no tardará en volverse a la situación de partida, que se prolongará a lo largo de esa centuria y la siguiente.

${ }^{18}$ Ese año, el Licenciado D. Antonio Vallejo Suárez ordenó en su visita que se compraran media docena de mantas y otras tantas sábanas y almohadas, tres colchones, dos asadores de hierro, un sartén, ollas y una docena de escudilla y platos. No sabemos si llegaron a efectuarse todas esas adquisiciones, pero tal menaje no aparece en el inventario que se elaboró en la visita siguiente, bien porque se deterioró o porque no llegó a cumplirse la orden. AHDA Libro de San Juan.

${ }^{19}$ Por ejemplo, cuando falleció D. Diego Tejeiro y Valcarce, entre 1621 y 1633, sus hermanos entregaron al hospital "un cama de ropa del difunto", que estaba compuesta 
Para la atención de los pobres que llegaba el centro contaba con una plantilla muy reducida. Durante los siglos XVII y buena parte del XVIII la formaban cuatro personas: una hospitalera, el mayordomo, un barbero y el capellán. La primera, además de mantener limpio el hospital y de hospedar a los que llamaban a su puerta, se encargaba del aseo de la ermita. Esa trabajadora fue desapareciendo de la contabilidad a lo largo de la centuria dieciochesca. Por su parte, el mayordomo gestionaba la economía del centro y el capellán debía de decir las misas semanales en la ermita, administrar los sacramentos a los "peregrinos" y enfermos y enterrar a los que fallecían.

Respecto a la labor hospitalaria, ambos ofrecían una variada gama de servicios, aunque las atenciones prioritarias eran bien distintas. El de la Reina tenía como objetivo preferente la atención médico-sanitaria a los enfermos allí alojados. De ésta se beneficiaban los pobres de solemnidad que no padecían enfermedades crónicas ${ }^{20}$. Por su parte, el de San Juan estaba orientado a cubrir las atenciones más básica de los peregrinos y transeúntes. Labor que no descuidó el de Ponferrada ${ }^{21}$, puesto que también ofrecía limosnas a los indigentes, generalmente en comida, daba cama a los peregrinos y pasajeros o realizaba labores de traslado ${ }^{22}$. Así mismo, ambos cubrían otra de las necesidades de los pobres: la atención espiritual. En ese momento la salud del alma ${ }^{23}$ era tan apreciada como la

por dos colchones de lana, dos mantas, dos almohadas de lana y dos sábanas". AHDA Libro de San Juan.

${ }^{20}$ Limitaciones para que ingresara pacientes de este tipo también se constatan en otros centros hospitalarios, caso, por ejemplo del de Santiago de Compostela. Vid. GARCÍA GUERRA, Delfín - El hospital Real de Santiago..., cit., p. 52.

${ }^{21}$ Esa multiplicidad de funciones asistenciales también se observa en otros hospitales de época medieval. MARTÍNEZ GARCÍA, Luís - La asistencia a los pobres en Burgos durante la Edad Media. El Hospital de Santa María la Real (1341-1500). Burgos: Diputación de Burgos, 1981, p. 26.

${ }^{22}$ Consistían éstas en transportar en caballo, hasta la etapa siguiente del viaje, a los enfermos ya curados o a aquellos que manifestaban mejoría. Les daban, aunque no siempre, una pequeña cantidad de dinero para que en los días inmediatos pudieran comprar alimento "para sacar a un pobre del hospital 3 reales y dos raciones para el camino son 4,5 reales". Estos traslados no fueron muy frecuentes en los años que disponemos de contabilidad, su número osciló entre los 4, de 1661, y los 15 del año anterior.

${ }^{23}$ A este respecto Vid. HENDERSON, John - Healing the body and saving the soul: hospitals in Renaissance Florence. Renaissance Studies". Journal of the Society for Renaissance Studies. 15-2 (2001) 188-216; VALENZUELA CANDELARIO, José -Hospitales y Beneficencia. Historias de hospitales. Revista de Trabajo Social y Salud. 43 (2002) 39-66. 
física y muchas de esas gentes, en constante trasiego y sin una referencia religiosa estable, no la tenían asegurada. Junto a estas ayudas más comunes, ambos podían ofrecer auxilios extraordinarios, como, por ejemplo, la lactancia de niños abandonados.

\section{Las bases materiales: procedencia de los ingresos y destino de los gastos}

La capacidad económica de los centros hospitalarios era lo que en último término determinaba su alcance benéfico y social. En el caso de los bercianos que nos ocupan, ninguno de los dos gozaba de un patrimonio importante, eran dos Obras Pías modestas, una más que otra. En el de la Reina el monto global de sus ingresos, durante la segunda mitad del siglo XVII y comienzos del XVIII, se situó entre los 2000-3000 reales ${ }^{24}$. Por su parte, los del hospital de San Juan pasaron de poco más de 500 reales, antes de 1705, a situarse, a partir de esa fecha y hasta finales de la década de los sesenta, en trono a los 330-360; experimentado en 1768 una nueva caída, debido a la dificultades para cobrar algunas rentas.

Las entradas de dinero del centro ponferradino a finales del siglo XVI, provenían, fundamentalmente, de dos partidas: un "juro" y las donaciones que semanalmente efectuaban las "personas ricas y principales" de la villa. En 1661 el ayuntamiento, consciente de las limitaciones y dificultades que tenía el hospital de la Reina para atender a los enfermos, le agregó las rentas del inactivo hospital de San Lázaro ${ }^{25}$, lo que le supuso ampliar su dotación económica en torno a un 12-13\%. La falta de un criterio uniforme

${ }^{24}$ Cifra calculada en base a las cuentas de los años y trienio siguientes: 1662, 1663, 1664, 1676-79, 1687-89, 1699, 1700, 1701, 1702 y 1721. AHMP Legajos de Cuentas del Hospital de la Reina.

25 "Los señores justicia y regimiento de esta villa como patronos del hospital de San Lázaro de esta villa, reconociendo el poco caudal de renta del hospital de la Reina de esta villa con que se falta al socorro de los pobres que vienen a dicho hospital, y por reconocer que en el de San Lázaro no los hay, agregaron la renta de dicho hospital al de la enfermería de esta villa". AHMP, Leg. 26. 
a la hora de elaborar las cuentas del hospital ${ }^{26}$ nos dificulta el poder realizar un seguimiento minucioso de la naturaleza de esos ingresos a lo largo de la Edad Moderna. No obstante, tomando como base una pequeña muestra del último cuarto del siglo XVII y principios del XVIII, en la que se efectuó un desglose anual más o menos detallado, podemos acercarnos a la procedencia de los mismos en ese momento, notablemente diversificada respecto a la centuria anterior. En torno al 50\% derivaban de las rentas generadas por los "censos", seguidas de las procedentes del patrimonio rústico. Dentro de éstas, cabe destacar las cuarenta "fanegas" que pagaba al hospital el cercano Monasterio de Carracedo por la extensa heredad del coto de Fabero ${ }^{27}$. Esta aportación representaba entre el 50 y el $60 \%$ del montante global de ese capítulo. Con porcentajes que oscilan entre el 5,4\% y $11,1 \%$, se registran las dimanadas del "juro" que había sido donado por Isabel la Católica en $1499^{28}$. Finalmente, apenas tuvieron importancia porcentual las limosnas, salvo en $1702^{29}$, o la venta de ropa de los pobres que fallecían ${ }^{30}$ (Gráfico n. ${ }^{\circ} 1$ ).

En el de Cacabelos las entradas de dinero procedían únicamente de dos partidas, los censos y las rentas que generaban sus bienes raíces. A través del inventario de patrimonio realizado en 1727, en el que se tomó como base

${ }^{26}$ Algunos años las cuentas aparecen muy resumidas y otros agrupadas por el periodo de mandato de los "mayordomos".

${ }^{27}$ La propiedad de esos terrenos condujo al hospital a un largo pleito, cuyo desenlace final tuvo lugar en los primeros años del siglo XIX, resultando desfavorable para el centro asistencial. AHMP, Leg. 26.

${ }^{28}$ Ese "juro" en un principio debía cobrarse sobre "la dehesa y herbaje del coto de Cabañas de Fabero". Sin embargo en 1566 ya se percibía "sobre las rentas de la villa de Ponferrada", y, a lo largo del XVII y comienzos del XVIII "sobre las alcabalas de esta villa y partido". AHMP Leg. 27.

${ }^{29}$ Estas dádivas, que hemos encontrado también en otros momentos en cantidades muy modestas, sólo en 1702 tuvieron un significado porcentual relativamente importante en el capítulo de ingresos. Se trataba de una cantidad destacada, 440 reales, procedentes de una manda testamentaria realizada por Dña. Antonia Gamarra, vecina de Ponferrada. AHMP Libros de Cuentas.

${ }^{30}$ Era ésta una práctica común en los establecimientos de este tipo. En nuestro caso desconocemos la importancia que tuvo este capítulo en las rentas del centro. Vid. GONZÁLEZ DÍAZ, Antonio Manuel - Poder urbano y asistencia social: el hospital de San Hermenegildo de Sevilla (1453-1837). Sevilla: Diputación de Sevilla, 1997, p. 212. Este tipo de costumbres llevó a Ramos Martínez. a considerar que el fallecimiento de un enfermo era más productivo para el hospital que el restablecimiento del mismo. RAMOS MARTÍNEZ, Jesús - La salud pública y el Hospital General de la ciudad de Pamplona. Pamplona: Gobierno de Navarra, 1989, p. 234. 


\section{Gráfico n. ${ }^{\circ} 1$ - Procedencia de los ingresos del Hospital de la Reina} (finales del S. XVII- principios del S. XVIII)

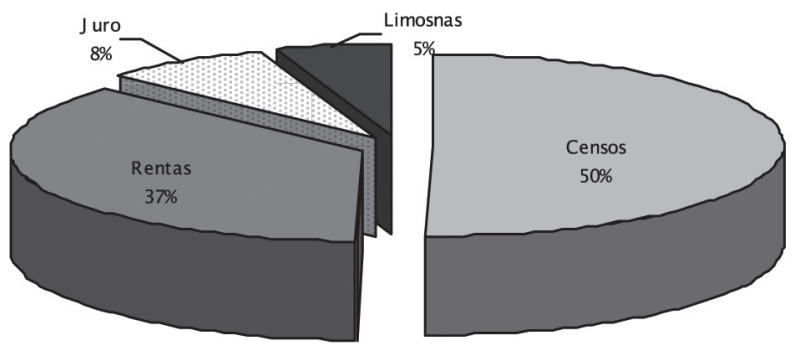

Fuente: A.H.M.P. Legajos de cuentas del Hospital de la Reina.

otro de 1696, sabemos que percibían: 50 cantaras de vino en concepto de foro sobre unos viñedos; 26 reales en metálico que les rentaban dos casas, otras tantas viñas, un horno y 5 tierras; y los réditos que les reportaban 738 ducados que tenían invertidos en 24 censos. En todos los casos de trataba de cesiones antiguas, pues si las escrituras de foro se habían formalizado todas ellas en 1643, los préstamos habían tenido lugar, mayoritariamente, en la primera mitad del siglo XVII. En este centro la dependencia económica de los censos aún era mayor que en el anterior, pues de los réditos generados por éstos recaudaban entre el 71\% y algo más del $90 \%$ de sus ingresos. Esa tónica se mantuvo a lo largo de toda la serie, y aún después de que en 1705 se rebajaran los tipos de interés. A continuación estaban las provenientes de la venta del vino, cuyos porcentajes estuvieron sometidos a oscilaciones, más o menos bruscas, dependiendo del precio de mercado. Por su parte, el cobro de las rentas en metálico del patrimonio rústico e inmueble, aunque en el Catastro del marqués de la Ensenada aún se le imputan, la realidad es que fueron perdiendo fuerza hasta desaparecer a medida que avanzaba el siglo XVIII. No sabemos si ese quebranto derivó de la desidia de los administradores o porque en algún momento se cambiaron por otro tipo de prestación. A tenor de lo que ocurrió en 1768, parece que la primera opción sea la más viable. En ese año se descargaron de los ingresos 228 reales de los intereses que cobraban por los censos y de otras rentas por "incobrables", simplemente señalan como causas expresiones tan genéricas como "se negó" o "se opuso" (Tabla n. ${ }^{\circ} 1$ ). 
Tanto en Ponferrada como en Cacabelos un mal endémico fue la mala administración económica. En el de la Reina, en las vistas realizadas al hospital en 1566 y 1687 recriminaban a los administradores que no efectuaban su trabajo con el celo requerido. En la primera, efectuada por el corregidor de la villa Ponferrada, se dejaba constancia de la desmembración del pequeño patrimonio del centro a causa de la mala gestión. Señalaba, entre otras deficiencias, que no se estaban cobrando los 7.000 "maravedíes" con los que había dotado la reina Isabel a la fundación o que no se anotaban, con la minuciosidad necesaria, las donaciones que semanalmente hacían los individuos más ricos de la localidad. Para corregir esa situación calamitosa ordenaba que a partir de entonces se hiciera un inventario con todos los bienes que poseía el centro y que se pidieran fianzas a los mayordomos, para que avalaran con su patrimonio las pérdidas que pudiera ocasionar una mala gestión. Por su parte, en la de $1687^{31}$ el representante del obispo de Astorga ${ }^{32}$ recriminaba a los responsables económicos que "no tomaron las cuentas en los últimos años". Esa inadecuada administración de las rentas parece que fue un mal frecuente en el centro y aún adquirió mayores dimensiones a finales del siglo XVIII. En 1787 se señalaba "el deplorable estado y mala administración de las rentas" como la causa principal, que justificaba por sí sola, la necesidad de elaborar un nuevo reglamento para el gobierno del citado hospital.

En el de Cacabelos la mala gestión tuvo, además, otra vertiente. El balance entre ingresos y gastos que se desprende de la muestra es generalmente de signo positivo, salvo en los cuatro años - 1626, 1675, 1716 y 1725 - en los que se llevaron a cabo obras de remodelación en el centro. La cifra media de ahorro, cuyo porcentaje se sitúa en el 39,5\%, de los ingresos, fue superada en varios años, llegando a alcanzar el $83,6 \%$ en 1753 . Esos porcentajes fueron mucho más bajos en Ponferrada, donde la global del periodo apenas

${ }^{31}$ Disponemos de otra visita, realizada en 1682, en la que no se hace ningún tipo de referencia a las cuestiones económicas, por lo que es de suponer que los mayordomos estuvieran cumpliendo meticulosamente sus funciones.

${ }^{32}$ Ese relevo en las actividades de supervisión del hospital de los responsables eclesiásticos frente a los civiles está visiblemente relacionado con las disposiciones del Concilio de Trento, en las que se facultaba a los obispos a visitar todos los centros de estas características. Vid. GONZÁLEZ DÍAZ, Antonio Manuel - Poder urbano y asistencia social..., cit., p. 61. 
alcanzó el 6\% y la más elevada de la muestra, que se constató en 1662, fue del 23\%. Los resultados obtenidos en San Juan podrían obedecer a una escrupulosa política de ajuste de caja o a una baja demanda de atenciones, que de hecho debió de ser así. Pero el que se reclamara a los mayordomos, prácticamente en todas las visitas pastorales, el reintegro de los alcances nos hace sospechar que estos hombres actuaron más en beneficio propio que al servicio del necesitado ${ }^{33}$, por supuesto con el consentimiento de los patronos. Es lógico pensar que dicha permisividad derivara de la confluencia, por un lado, de una baja demanda de asistencias, que al no ocasionar una presión sobre el centro tampoco generaba la vigilancia constante de los máximos responsables; y, por otro, que al ser el cargo de mayordomo de renovación anual, recayendo antes o después en todos los vecinos de la localidad, la transigencia entre ellos debió de ser la nota dominante.

Respecto a la distribución del gasto en estos centros asistenciales, en Ponferrada comprobamos como los dos conceptos que más dinero absorbían eran las raciones de los enfermos ${ }^{34} \mathrm{y}$ los salarios del personal (Gráfico n. ${ }^{\circ}$ 2). En conjunto acapararon algo más del 50\% de las inversiones, situándose en 1662, 1663 y 1664 por encima del 75\%. La representación porcentual de los primeros era muy variable, pues dependía del número de demandas

Gráfico n. ${ }^{\circ} 2$ - Distribuión de los gastos del Hospital de la Reina de Ponferrada (1662-1698)

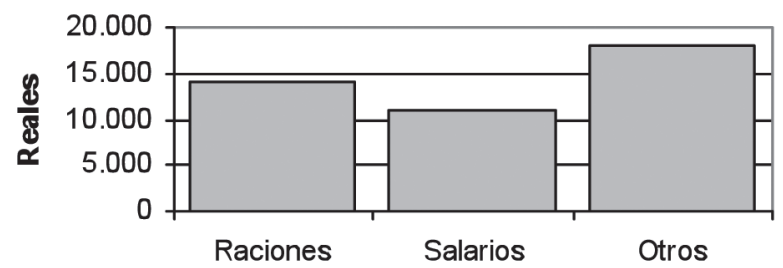

Fuente: A.H.M.P. Legajos de cuentas del Hospital de la Reina.

${ }^{33}$ Las referencias a esas deudas son constates en las visitas, por ejemplo, en 1767 el visitador D. Manuel Díaz Quijano realizó un recuento de alcances y el monto global de lo adeudado ascendía a 8.848 reales, de los cuales 5.435 se habían acumulado entre 1686 y 1748, y el resto desde esa última fecha hasta 1767. AHDA Libro de San Juan.

${ }^{34}$ La importancia porcentual que en los gastos tenían los alimentos de los enfermos parece que es frecuente en otros centros. Vid. GARCÍA HOURCADE, José Jesús - Beneficencia y sanidad en el S. XVIII. El hospital de San Juan de Dios de Murcia. Murcia: Universidad de Murcia, 1996, p. 117. 
Cuadro ${ }^{\circ} 1$ - Cuentas del Hospital de San Juan de Cacabelos

\begin{tabular}{|c|c|c|c|c|c|c|c|}
\hline & Ingresos & Gastos & Alcance & & Ingresos & Gastos & Alcance \\
\hline 1626 & 317 & 451 & -134 & 1721 & 333 & 245 & 88 \\
\hline 1659 & 789 & 464 & 325 & 1723 & 324 & 240 & 84 \\
\hline 1675 & 544 & 922 & -378 & 1724 & 333 & 280 & 53 \\
\hline 1702 & 561,5 & 255 & 306,5 & 1725 & 333 & 369 & -36 \\
\hline 1703 & 591 & 306 & 285 & 1726 & 405 & 216 & 189 \\
\hline 1704 & 590 & 261 & 329 & 1734 & 360 & 228 & 132 \\
\hline 1705 & 366 & 305 & 61 & 1735 & 365 & 190 & 175 \\
\hline 1706 & 364 & 285 & 79 & 1739 & 403 & 211 & 192 \\
\hline 1707 & 354 & 273 & 81 & 1742 & 329 & 185 & 144 \\
\hline 1708 & 352 & 233 & 119 & 1743 & 377 & 187 & 190 \\
\hline 1709 & 364 & 199 & 165 & 1744 & 345 & 172 & 173 \\
\hline 1710 & 366 & 211 & 155 & 1753 & 329 & 54 & 275 \\
\hline 1713 & 364 & 155,5 & 208,5 & 1759 & 330 & 207 & 123 \\
\hline 1714 & 326 & 296 & 30 & 1761 & 330 & 192 & 138 \\
\hline 1716 & 329 & 716 & -387 & 1765 & 330 & 203 & 127 \\
\hline 1717 & 314 & 185 & 129 & 1768 & 186 & 102 & 84 \\
\hline 1720 & 334 & 264,5 & 69,5 & 1769 & 124 & 201,5 & $-77,5$ \\
\hline
\end{tabular}

Fuente: A.H.D.A. Libro de San Juan de Cacabelos.

de asistencia: así, por ejemplo, en el último trimestre de 1677 el hospital no recibió a ningún pobre. Por el contrario, en los salarios se observa una cierta estabilidad, pues se constatan variaciones mínimas entre 1676 y 1702 . El resto de las partidas que conforman los gastos son de lo más variado: leña, jabón, velas, medicinas, traslado de enfermos, celebraciones religiosas, enseres para el hospital, reparaciones, etc. Precisamente estas dos últimas - reposición de ajuar y menaje para el acomodo de enfermos y arreglos del hospital y capilla de San Lázaro - se intensificaron en el último cuarto del siglo XVII ${ }^{35}$ y en 1702.

${ }^{35}$ Así, por ejemplo, entre 1687 y 1689 se llevaron a cabo lo que las fuentes denominan “obra nueva en el hospital", que supuso una inversión de 2.044,7 reales. En esas mismas fechas también se compró ajuar por valor de 263,7 reales. Unos años más tarde, en 1697 , se realizaron obras en la fachada del hospital. Por su parte en 1702, se reparó la techumbre de San Lázaro, por un coste de 340 reales, gastándose otros 198 en la reposición de ropa para las camas. Todo ello fue posible gracias al pequeño remonte en la partida de ingresos. AHMP Libros de Cuentas. 
Para conocer la distribución de gastos en San Juan hemos optado por agruparlos en tres conceptos: espirituales, hospitalarios y otros (Gráfico n. ${ }^{\circ} 3$ ). En el primero hemos incluido los destinados a pagar los actos religiosos que se celebraban el día del patrono y las misas semanales. El segundo, que es el más amplio, abarca los libramientos de los pobres y el costo de conducir enfermos a otros centros mejor dotados, generalmente a Villafranca del Bierzo y esporádicamente a Ponferrada; los salarios de las hospitalera y cirujano; el entierro de los pobres que allí fallecieron; la compra de leña, paja, jabón, menaje, ajuar y las inversiones en el remozado del edificio. Finalmente, el genérico otros contiene los gastos que les ocasionaba el hacer las cuentas o las visitas pastorales y aquellos extraordinarios que no tienen cabida en ninguno de los anteriores. Este último bloque fue excepcionalmente elevado en 1659 y 1675 , debido a que sufragaron la crianza de un expósito.

Gráfico n..$^{\circ} 3$ - Distribución de los gastos del hospital de Cacabelos (1626-1772)

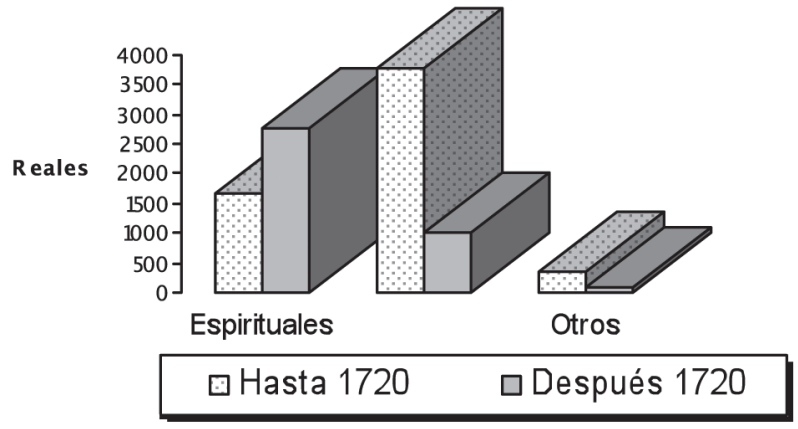

Fuente: A.H.D.A. Libro de San Juan de Cacabelos.

Respecto al reparto porcentual del gasto, podemos dividir la serie en dos bloques, cuyo punto de inflexión está en 1720. Hasta esa fecha, salvo el bienio 1702-1703, las mayores inversiones se realizaron en el funcionamiento del hospital; y a partir de ese momento, y también con alguna excepción, como 1725, fueron las actividades religiosas las que más dinero consumieron. Dentro del primer bloque, el mayor gasto no se encaminó precisamente a la atención de los pobres, pues, salvo 1626 o 1659, la mayor parte de los años sólo se consumieron con ellos en torno a los 30-40 reales, 
descendiendo a 11, e incluso a 3 en varios momentos. Los que más capital acapararon fueron los salarios y el abasto de leña y paja. Por su parte, en las ceremonias religiosas tuvo lugar un repunte en 1702 y 1703, debido a los festejos del patrono del hospital, San Juan. Al año siguiente se intentó corregir ese dispendio "atendiendo a la pobreza del centro", pero volvería a repetirse en 1708 y 1710 .

En la segunda parte de la serie, es decir a partir de 1720, el gasto hospitalario pasó a un plano secundario, y la mayor parte de los años se nutren exclusivamente de la adquisición de productos como el jabón, la cera o el aceite, que también eran consumidos por la ermita. De nuevo, hubo un incremento en la partida dedicada a la celebración del patrono, que pasará a situarse en los mismos niveles que tenía a comienzos del siglo XVIII.

\section{Las asistencias hospitalarias}

Conocer el nivel ocupacional de estos centros no ha sido tarea fácil. En el Hospital de la Reina hemos tenido que recurrir a una fuente indirecta - las raciones servidas - ya que no disponemos de registros de entradas o salidas, a pesar de la obligación que tenían los administradores de anotar en sendos libros esos movimientos, al menos desde el reglamento de 1788 . El problema del empleo de los listados de raciones como fuente alternativa radica en la sobrevaloración de los resultados. Pensemos que en estos registros toda persona que permaneciera ingresada en el centro más de un día, circunstancia bastante habitual, aparece contabilizada tantas veces como días duró su convalecencia ${ }^{36}$. Para subsanar en lo posible esta carencia de la fuente hemos optado por calcular la media de raciones servidas por día, ya que este indicador nos proporciona la media ocupacional diaria.

${ }^{36}$ En los hospitales de San Antonio Abad de la ciudad de León y San Juan de la ciudad de Astorga - ambos ubicados en la misma provincia que el de la Reina - el período medio de convalecencia oscilaba entre los veinte y treinta días. MARTÍN GARCÍA, Alfredo y PÉREZ ÁLVAREZ, María José - Hospitalidad y asistencia en la provincia de León a finales del Antiguo Régimen (1728-1896), Dynamis. 27 (2007), 157-185 (p. 179); MARTÍN GARCÍA, Alfredo - Pobres y enfermos en el León de la Edad Moderna: la asistencia hospitalaria en la ciudad de Astorga, in RUBIO PÉREZ, Laureano M. (Ed.) - Pobreza, marginación y asistencia en la Península Ibérica (siglos XVI-XIX), León: Universidad de León, 2009, p. 65-97. 
Así, por ejemplo, para el año 1661 las 1.248 raciones repartidas suponían unas 3,4 atenciones por día.

Tomando como base ese índice podemos comprobar, en primer lugar, el importante crecimiento cuantitativo de las asistencias en el siglo XVIII, en comparación con la centuria precedente (Gráfico n. ${ }^{\circ}$ 4). De hecho, su número llegó a duplicarse, pasando de entre dos y cuatro dietas diarias a situarse por encima de las siete. Esa tendencia al alza continuó a mediados de siglo y se acentuó con fuerza en el último cuarto, momento de aguda crisis a nivel provincial y a la que respondió el centro ampliando su oferta ocupacional. Es muy posible, aunque la fuente no ofrece ya más datos, que esa tónica alcista de las asistencias aún continuase durante los primeros años del XIX, como se ha podido observar para las vecinas ciudades de Astorga y León. A ese incremento contribuirían significativamente los pacientes forasteros. Nos referimos tanto a los transeúntes y peregrinos que

Gráfico n. ${ }^{\circ} 4$ - Número medio de asistencias diarias ofrecidas por el Hospital de la Reina (1659-1800)

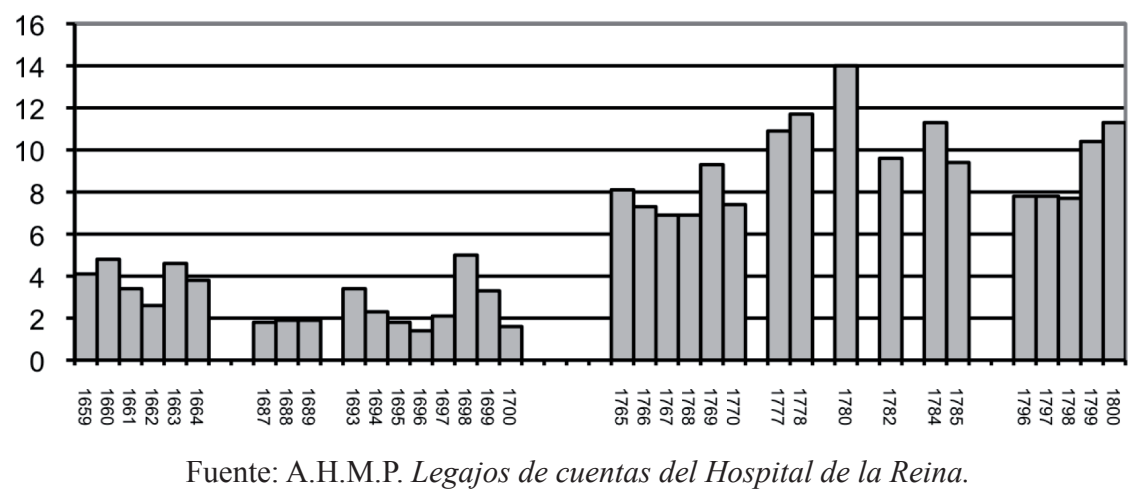

deambulaban por el Camino de Santiago como a los vecinos del entorno rural más próximo. Estos últimos, en momentos de dificultades económicas acudían a Ponferrada, como cabecera de la comarca, buscando la ayuda de sus instituciones caritativas.

Un capítulo importante en hospitales como el de la Reina, orientado a la atención de los pobres, era el de la alimentación que recibían los enfermos, elemento terapéutico de primer orden para el restablecimiento muchos de 
ellos. La información de la que disponemos para conocer esta vertiente de la asistencia es muy puntual. Para finales del siglo XVII y comienzos del XVIII disponemos algunas referencias que nos han permitido saber que en esos momentos se elaboraba en la cocina del centro un "puchero de los pobres", a base de garbanzos y tocino y condimentado con azafrán y otras especias. Además de esos alimentos básicos, se anotó la compra de carnero, y, excepcionalmente ${ }^{37}$, de miel, azúcar, huevos, leche, bizcocho, pan, vino o aguardiente. Las noticias relativas a las dietas son más abundantes a medida que discurre la centuria dieciochesca y, de hecho, hemos podido saber que entre 1765 y 1769 la ración ofrecida a los enfermos incluía unos 400 gramos de pan, acompañado de vaca o carnero y vino. Junto a estos productos se generalizó el consumo de huevos, bizcocho, azúcar y chocolate ${ }^{38}$, aunque suponemos que al igual que en otros establecimientos estos alimentos estaban destinados a regímenes especiales. Más detallados son los resúmenes mensuales de consumo alimentario en el año1800. Las raciones diarias de los convalecientes consistían, aproximadamente, en 362 gramos de carne, 453 de pan, 45 de tocino, 68 de garbanzos y 0,8 de manteca de cerdo, acompañado todo ello de un cuarto de litro de vino. Si era necesario, esa dieta se complementaba con los productos ya reflejados en la documentación de la centuria anterior - bizcocho, chocolate, castañas, leche o azúcar ${ }^{39}$ o con otros nuevos, caso del arroz. Por el contrario el consumo de huevos, importante en los años anteriores, pasó a ser ocasional ${ }^{40}$.

${ }^{37}$ Por ejemplo, en el caso de la miel en 1694 solamente se compró medio litro en el mes de diciembre o uno en 1699. En marzo de 1696 se gastó en bizcocho, azúcar, huevos y especias tan solo 5 reales. AHMP Libros de Cuentas.

${ }^{38}$ El chocolate en ese momento debemos considerarlo un producto medicinal. Vid CORONAS TEJADA, Luís - El hospital Real de Nuestra Señora de la Misericordia..., cit., p. 85 .

${ }^{39}$ A tenor de los productos adquiridos, la dieta ofrecida a los enfermos del hospital de Ponferrada era muy similar a la que recibían los de Loja o Pamplona. Vid CORONAS TEJADA, Luís - El hospital Real de Nuestra Señora de la Misericordia,..., cit., p. 83-87. RAMOS MARTÍNEZ, Jesús - La salud pública y el Hospital General..., cit., p. 324 y ss.

${ }^{40}$ Así, por ejemplo, en mayo de 1796 o en junio de 1800, se compraron solamente dos huevos. En el primer caso los administradores precisaron que se destinaban para "hacer gárgaras" y en el segundo "para un enfermo de digestivo". El que prácticamente desaparezcan los huevos en el consumo hospitalario puede estar reflejando la crisis en que se veía envuelta la provincia de León desde los últimos años del siglo XVIII. Lógicamente esa afectaría a la institución, cuyas rentas se veían mermadas por falta de pago de los colonos. En el libro 
Para San Juan Bautista las referencias cuantitativas que poseemos, referentes a la demanda asistencial, son aún más indirectas. Tomando como base el gasto que la atenciones representaban en las cuentas, podemos concluir que la demanda era muy baja. Otros indicadores, como el número de fallecidos en el hospital o los traslados efectuados a otros centros, apuntan en la misma dirección. En la serie de cuentas que hemos logrado reunir tan sólo en 10 años aparece el traslado de uno o a lo sumo dos pobres a otra localidad, salvo en el crucial año de 1710 en que se condujeron 10 a Villafranca. Respecto a los óbitos, únicamente se constatan en 5 años, y en conjunto no llegaron a la decena.

Por otro lado, a partir de mediados de la década de los treinta del siglo XVIII comienzan a ir desapareciendo de la documentación económica los cargos más directamente conectados con la asistencia. La ausencia más destacada es el salario de la hospitalera, no obstante, el que algún año se constate el pago al barbero y una pequeña propina a una mujer nos hacen pensar en asistencias muy puntuales. Así mismo, en la visita que realizó D. Manuel Díaz Quixano, en 1767, ordenaba al mayordomo que contribuyera con lo necesario para la manutención de los enfermos y al cura ${ }^{41}$ que velara porque el mandato se ejecutase. Parece que la asistencia en ese momento, sino inexistente, sí al menos estaba muy descuidada, pues ya hacia años que en la contabilidad no se imputaban partidas por este concepto. Así mismo, en atención a la cortedad de las rentas del centro, y dando por incobrables las deudas contraídas con él, decidió que a partir de entonces sólo se admitieran

de acuerdos se recoge, el 26 de julio de 1800: "debido a la esterilidad de la cosecha los colonos no pueden pagar la renta”. AHMP Leg. 26. Respecto al papel que representaban los huevos en las fluctuaciones económicas, Vid. BRAUDEL, Fernand - Civilización material, economía y capitalismo (s. XV-XVIII), vol. 1. Madrid: Alianza, 1984, p. 174. Por su parte sobre la incidencia de las coyunturas económicas en la acción benéfica, Vid. CARASA SOTO, Pedro - Pauperismo y revolución liberal en Burgos (1750-1900). Valladolid: Universidad de Valladolid, 1987, p. 378.

41 “...y aunque este cargo tal vez le pueda parecer molesto, considera justamente su merced es anejo al ministerio que ejerce en usar de caridad, visitando a los enfermos y cuidar se les suministre lo preciso al restablecimiento de sus enfermedades...". AHDA Libro de San Juan. 
a personas que estuvieran realmente enfermas, siendo desviados el resto de los que allí recabaran a la localidad de Villafranca del Bierzo ${ }^{42}$.

\section{Conclusión}

En la comarca berciana a finales de la Edad Media y comienzos de la Moderna tuvo lugar una reestructuración de la red hospitalaria a instancias de la Corona. Al margen de esa existió otra paralela que afectó, en mayor o menor grado, a los centros que no se adaptaron a las nuevas circunstancias. De los quince que sobrevivieron, sólo el de Santiago, en Villafranca del Bierzo, y el de la Reina pueden ser considerados hospitales con la acepción que actualmente tiene ese vocablo.

El de la Reina, que si bien es cierto que ya renació con una vocación sanitaria, tal dimensión lo convirtió en punto de referencia para los necesitados comarcanos y forasteros y, posiblemente, esa demanda fue el motor de presión para mantenerlo operativo. No obstante, y a pesar de que sus pequeñas dimensiones y su reducido patrimonio, por otra parte frecuentemente mal gestionado, restringían notablemente su acción social, tuvo una notable importancia en una comarca geográficamente aislada, sin peso urbano y muy transitada.

En el caso opuesto, y a pesar de que sus rentas le hubieran permitido cubrir una mayor demanda, tenemos el de San Juan, que fue languideciendo hasta prácticamente desaparecer en la segunda mitad del siglo XVIII. Posiblemente, ese mismo destino fue el que corrieron otros centros similares a él y también los peor dotados. El móvil religioso de obediencia a los mandatos divinos, que había sido origen de todas esas fundaciones y de su mantenimiento, ya no tenía por si solo fuerza para dinamizar ese tipo de infraestructuras de atención a los necesitados.

42 “...puesto que distando esta villa una legua de Villafranca y que en ella hay hospital bien surtido y opulento en el que se admite a todo peregrino o transeúnte...", AHDA Libro de San Juan. 